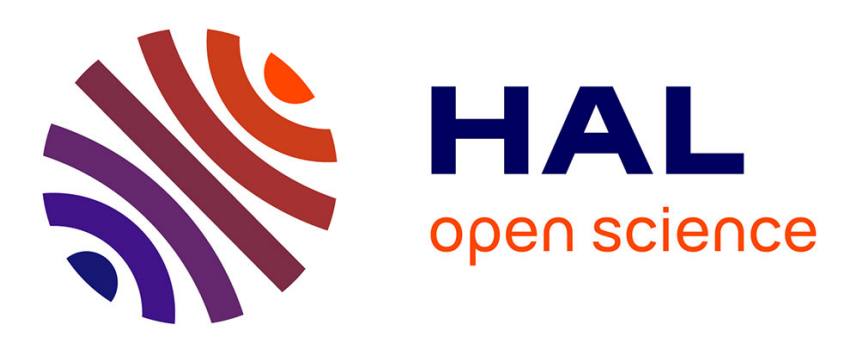

\title{
Wavelength dependence of nonlinear circular dichroism in a chiral ruthenium-tris(bipyridyl) solution
}

\author{
H. Mesnil, Marie-Claire Schanne-Klein, François Hache, M. Alexandre, G. \\ Lemercier, Chantal Andraud
}

\section{- To cite this version:}

H. Mesnil, Marie-Claire Schanne-Klein, François Hache, M. Alexandre, G. Lemercier, et al.. Wavelength dependence of nonlinear circular dichroism in a chiral ruthenium-tris(bipyridyl) solution. Physical Review A: Atomic, molecular, and optical physics [1990-2015], 2002, 66 (1), pp.138021. 10.1103/PhysRevA.66.013802 . hal-00837032

\section{HAL Id: hal-00837032}

https://hal-polytechnique.archives-ouvertes.fr/hal-00837032

Submitted on 19 May 2014

HAL is a multi-disciplinary open access archive for the deposit and dissemination of scientific research documents, whether they are published or not. The documents may come from teaching and research institutions in France or abroad, or from public or private research centers.
L'archive ouverte pluridisciplinaire HAL, est destinée au dépôt et à la diffusion de documents scientifiques de niveau recherche, publiés ou non, émanant des établissements d'enseignement et de recherche français ou étrangers, des laboratoires publics ou privés. 


\title{
Wavelength dependence of nonlinear circular dichroism in a chiral ruthenium-tris(bipyridyl) solution
}

\author{
H. Mesnil, M. C. Schanne-Klein, and F. Hache* \\ Laboratoire d'Optique et Biosciences, CNRS/INSERM/ENSTA/École Polytechnique, 91128 Palaiseau Cedex, France \\ M. Alexandre, G. Lemercier, and C. Andraud \\ École Normale Supérieure de Lyon, STIM, 69364 Lyon Cedex 07, France
}

(Received 19 October 2001; published 8 July 2002)

\begin{abstract}
Nonlinear circular dichroism is studied in a solution of ruthenium-tris(bipyridyl) salt in one-beam and pump-probe experiments by tuning the laser wavelength across the circular dichroism structure. The dispersion of the nonlinear circular dichroism is measured. This wavelength dependence is well accounted for by a model calculation where nonlocality is included in the optical response of a two-coupled-oscillator system. This calculation also allows us to address the question of the contribution of electric quadrupolarization to the nonlinear optical activity of an isotropic liquid of chiral molecules.
\end{abstract}

DOI: 10.1103/PhysRevA.66.013802

PACS number(s): 42.62.Fi, 33.55.Ad, 42.65.An

\section{INTRODUCTION}

Chiral molecules that exist under two mirror-symmetrical configurations (enantiomers) are known to play an important role in biochemistry and therefore deserve a great deal of attention from physicists. Linear optical properties have been widely used, and optical activity (polarization rotation or circular dichroism) has proved to be a very sensitive probe of chirality, but the application of nonlinear optics has emerged only recently for the study of chiral molecules. Although third-order nonlinear effects were predicted more than 30 years ago [1,2], only a few nonlinear optical rotation experiments were undertaken in the last decade $[3,4]$. In a recent paper [5], we demonstrated experimentally the existence of a nonlinear circular dichroism in a chiral rutheniumtris(bipyridyl) salt by measuring the variation of the circular dichroism (CD) as a function of the light intensity and observing that this $\mathrm{CD}$ decreases when the light intensity increases. This effect was shown to be in accordance with a theoretical calculation [6] predicting a saturation of the $\mathrm{CD}$ for a molecule described by a two- or three-level system. This experiment demonstrated the relevance of third-order nonlinear optics to study chiral molecules. In particular, it opened up a large field of investigation, as the optical Kerr effect is known to be a powerful technique in time-resolved spectroscopy. Following this first experimental demonstration, we extended this work to a degenerate pump-probe configuration and observed a pump-induced circular dichroism in the same ruthenium sample [7], again in agreement with theoretical expectations. All these experiments were however based on measurements at a unique wavelength; in this paper, we examine the dependence of CD on wavelength.

In the following section, we present two sets of experiments in the ruthenium sample. In a first set, we measure the nonlinear CD induced by a unique beam as a function of the wavelength for a tuning range (447-483 $\mathrm{nm}$ ) spanning a large part of the $\mathrm{CD}$ structure, whereas, in the second one,

\footnotetext{
*FAX: (+33)1-69-33-30-17. Email address: francois.hache@polytechnique.fr
}

we use a pump-probe configuration with a probe tunable from 410 to $490 \mathrm{~nm}$ covering the whole CD spectrum. In both cases, we resolved, for the first time to the best of our knowledge, the dispersion of the nonlinear circular dichroism. In order to describe our results, we develop in Sec. III a simple model, based on two two-level systems, which accounts well for the experimental CD spectrum of our sample. By expanding the electromagnetic field in terms of molecular extension in the nonlinear response calculation, we are able to obtain in a direct manner the expressions of the nonlocal contributions responsible for the chiral effects including linear as well as nonlinear terms. A very good agreement is found between this calculation and the experimental results, showing that we have a quite good understanding of the origin of the nonlinear circular dichroism in our sample. This calculation supports the evidence that the nonlinear optical activity we observe originates mainly from a nonlinear electronic response as a result of the saturation of the absorption.

In this calculation, the introduction of nonlocality is somewhat phenomenological and does not allow us to separate magnetic dipolar contributions from electric quadrupolar ones. We address this question in Sec. IV. Separating the electric quadrupolar nonlocal terms from the magnetic dipolar ones in our calculation, we show that even though the quadrupolar effects cancel out for two-level systems as previously shown [6], this cancellation is not general and we demonstrate their existence for anharmonic oscillators. This demonstrates that, as expected from general theoretical considerations [2], electric quadrupolarization can contribute to the nonlinear optical activity of isotropic liquids.

\section{EXPERIMENTAL RESULTS}

\section{A. One-beam experiment}

The sample is a ruthenium(II)-tris(bipyridyl) salt (RuTB) which exists under two enantiomeric forms, denoted $\Lambda$ and $\Delta$. The molecule structure is depicted in the inset of Fig. 1. The optical transition we are interested in is a metal-toligand charge transfer (MLCT) which gives rise to an ab- 


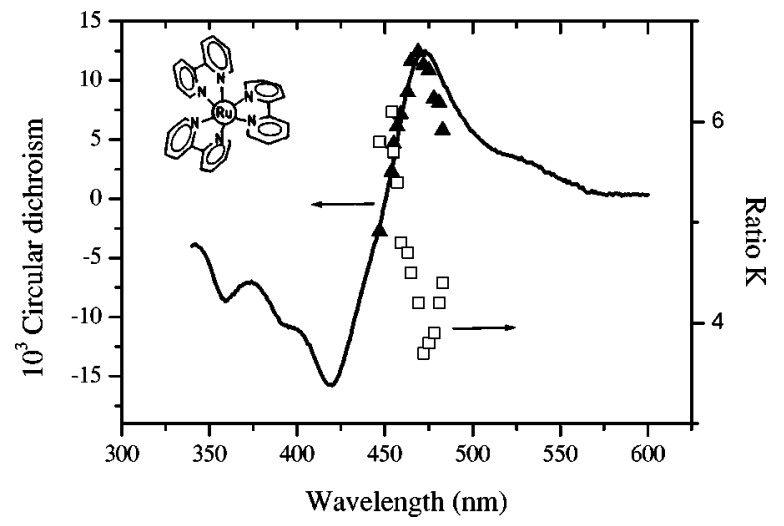

FIG. 1. Experimental determination of the circular dichroism (solid triangles) and of the ratio $K$ (open squares) obtained in the NLCD measurements in our RuTB samples. The solid line is the spectrum obtained with a CD spectrometer. The cell thickness is 2 $\mathrm{mm}$. Inset: structure of the ruthenium(II)-tris(bipyridyl) ${ }^{2+}$ ion.

sorption band in the blue and a bisignate CD structure (see Fig. 1). Previous experiments on this molecule [5,7] have shown that it behaves like a model molecule for which most of the third-order (Kerr) nonlinear response comes from the saturation of two-level systemlike transitions. Purely chiral effects were found in a one-color experiment where an intensity-dependent CD was observed [5]. The experimental setup that we use to study wavelength effects in the chiral nonlinear response is very similar to this one-beam experiment: a photomultiplier tube (PMT) measures the transmission of a laser beam through a solution $1.5 \times 10^{-3} \mathrm{M}$ of RuTB in ethanol placed in a circulating cell. The polarization of the laser beam is varied from left to right circular at $80 \mathrm{~Hz}$ by a longitudinal Pockels cell. This modulation allows us to extract from the PMT signal the chiral response through a lock-in (LI) detection technique and to precisely measure the $\mathrm{CD}$. The experiment then consists in measuring the $\mathrm{CD}$ as a function of the light intensity to observe purely chiral effects in the nonlinear response of the samples. The major difference compared to the former experiment is in the light source we utilize, since the former source did not allow the wavelength to be tuned across a significant part of the CD structure. The beam in the present study is derived from a sophisticated titanium-sapphire based femtosecond source from which we extract $150 \mathrm{fs}$ pulses tunable around $1.1 \mu \mathrm{m}$ at $1 \mathrm{kHz}$ repetition rate [8]. Performing sum-frequency generation of these pulses with $800 \mathrm{~nm}$ pulses originating from the primary laser in a 2-mm-thick-BBO crystal (type I), we obtain $180 \mathrm{fs}$ pulses tunable from 446 to $483 \mathrm{~nm}$. This tuning range allows us to span the red part of the $\mathrm{CD}$ spectrum. The pulse energy is in the microjoule range, low enough to restrict the nonlinear response to the third-order and to avoid thermal effects.

The following experimental procedure is repeated for all the wavelengths. After very careful alignment of the Pockels cell and of the various optical elements, we measure the PMT and the LI signals as a function of the intensity and deduce the $\mathrm{CD}[\propto(\mathrm{LI}) /(\mathrm{PMT})]$. This measurement is performed on the two enantiomers as well as on the racemic mixture to ensure that our setup is free of artifacts: we check that the CD's are symmetrical for the two enantiomers and null for the racemic, independent of intensity. When plotting $\mathrm{CD}$ vs incident intensity, we obtain straight lines from which we can extract two pieces of information [5]: the value at zero intensity gives the usual $\mathrm{CD}$ while the slope allows the determination of the chiral nonlinear response through a coefficient $K$ defined as follows.

Let us recall how we account for the saturation of the absorption in the moderate intensity regime we are working with: one writes the evolution of the light intensity $I$ along the $z$ direction as

$$
\frac{\partial I}{\partial z}=-(\alpha+\beta I) I
$$

where $\alpha$ is the linear absorption and $\beta$ accounts for the nonlinear response. In the case of a saturation of the absorption, $\beta$ is negative. For chiral molecules, both $\alpha$ and $\beta$ are expected to be different for the two handednesses of the light and we write

$$
\begin{aligned}
& \alpha=\alpha_{0} \pm \Delta \alpha, \\
& \beta=\beta_{0} \pm \Delta \beta,
\end{aligned}
$$

where the "plus" (minus) sign corresponds to the left (right) circular polarization for a given enantiomer. $\Delta \alpha$ is directly connected to the $\mathrm{CD}$ while $\Delta \beta$ describes the chiral nonlinear response, responsible for the nonlinear circular dichroism (NLCD) already observed [5]. The factor $K$ that we determine directly from the experimental curves is defined as

$$
K=\frac{\Delta \beta / \beta_{0}}{\Delta \alpha / \alpha_{0}} .
$$

Note that this parameter that gives the relative magnitude of the NLCD, is a molecular parameter independent of the sample concentration or of the light intensity. If the absorption and the $\mathrm{CD}$ involve the same transitions, it is also independent of such parameters as the oscillator strength or the lifetime of the excited states, but depends only on the energy level configuration [5]. It is therefore more readily comparable to experiments than $\Delta \beta / \beta_{0}$ which depends on a whole set of parameters.

The experimental results are plotted in Fig. 1. First of all, we note that the CD's we measure from the signal at low intensity as a function of the wavelength match the curve obtained with a CD spectrometer as expected. Concerning the ratio $K$, one can see that there is a strong variation of this parameter with the wavelength, with a clear correlation with the linear $\mathrm{CD}: K$ is minimum when the $\mathrm{CD}$ is maximum. The values we measure, around 4 , are in agreement with our previous one-color experiment [5].

\section{B. Pump-probe experiment}

In order to fully exploit the potentialities of the Kerr techniques, we have also developed a pump-probe experiment. In [7], we reported such an experiment in a degenerate configuration where the pump and the probe were derived from the 
same beam. This experiment has allowed us to demonstrate the existence of a pump-induced circular dichroism in a solution of the above-mentioned RuTB salt. Here, we extend this experiment to the case where the probe is continuously tunable whereas the pump is fixed at $465 \mathrm{~nm}$ (close to a maximum in the $\mathrm{CD}$ spectrum). To obtain the tunable probe, we pick up a part of the $800 \mathrm{~nm}$ pulses used in the generation of the pump beam, double its frequency and generate a white-light continuum by focusing this $400 \mathrm{~nm}$ beam in a sapphire plate. After filtering the $400 \mathrm{~nm}$ frequency, we obtain a pulse whose spectrum is almost flat from 410 to 490 $\mathrm{nm}$. The pulse energy is in the nanojoule range and the time duration, measured by cross correlation with the pump in a 1-mm-thick BBO crystal, was estimated to be 400 fs.

The setup is similar to the one described in [7]. The important point that we recall here is that the polarization of the probe is modulated from left to right circular while the pump is kept linearly polarized. Similarly to the one-beam experiment, this polarization modulation allows us to measure the $\mathrm{CD}$. The principle of the experiment is then to measure this $\mathrm{CD}$ as a function of the delay between the pump and the probe. The zero delay is straightforwardly determined by the time evolution of the PMT signal which displays a very clear jump when the pump and the probe overlap. The transmission change and the induced $\mathrm{CD}$ do not change in time on a few picosecond range [7]. Introducing the nonlinear coefficient $\beta_{p-p}$ similarly to Eq. (2.1), we can define the pumpinduced nonlinear absorption $\mathcal{A}$ as a function of the probe frequency $\omega$,

$$
\mathcal{A}(\omega)=\beta_{p-p}(\omega) I_{p}
$$

where $I_{p}$ is the pump intensity. The subscript $p-p$ is intended to differentiate this coefficient from its one-beam counterpart: $\quad \beta \propto \chi^{(3)}(\omega,-\omega, \omega) \quad$ whereas $\quad \beta_{p-p} \propto \chi^{(3)}(\omega$, $\left.-\omega_{\text {pump }}, \omega_{\text {pump }}\right)$. In the same way, we write

$$
\mathcal{D}(\omega)=\Delta \beta_{p-p}(\omega) I_{p}
$$

for the pump-induced $\mathrm{CD}$. The experimental values of the nonlinear absorption $\left(\beta_{p-p}\right)$ and $\mathrm{CD}\left(\Delta \beta_{p-p}\right)$ coefficients are plotted in Fig. 2 together with the linear absorption and $C D$ recovered from these measurements. The values are given in absolute units $\left(\mathrm{W}^{-1} \mathrm{~cm}^{2}\right)$ with a precision of $\pm 30 \%$ (due to the uncertainty in the pump energy and in the geometrical factors). We did not try to obtain more accurate values since this uncertainty factors out of the ratio of the two nonlinear coefficients. Looking at Fig. 2(a), one can deduce that the absorption band is composed of two different bands and we mainly saturate the low-energy one with the $465 \mathrm{~nm}$ pump. This translates in Fig. 2(b) by a dissymmetrical nonlinear CD spectrum, where again the induced effect is stronger on the low-energy side of the CD spectrum. Note that Fig. 2(b) is, to the best of our knowledge, the first third-order nonlinear $\mathrm{CD}$ spectrum ever measured.

For a more quantitative comparison with theory, we introduce the ratio $K_{p-p}$ which we extract from our data as

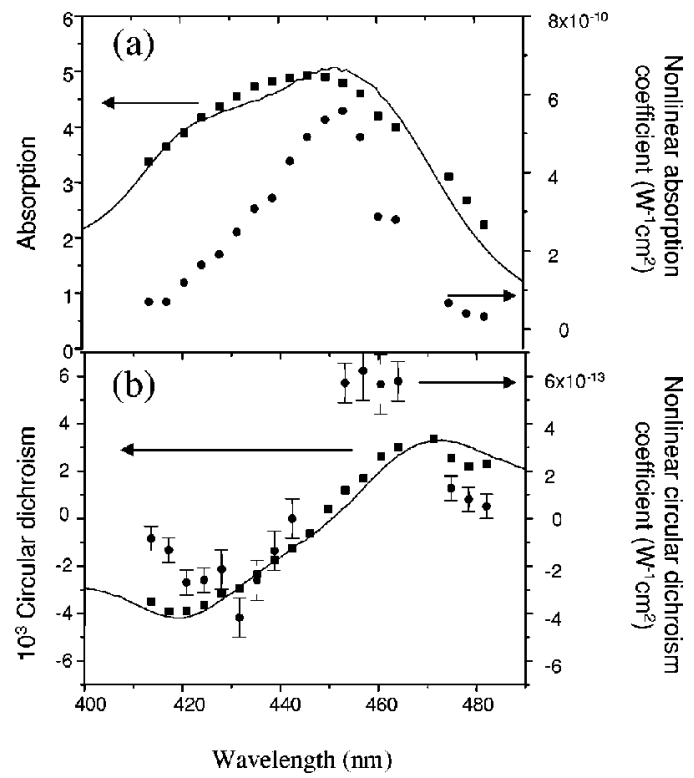

FIG. 2. (a) Linear (squares) and nonlinear (dots) absorption, (b) linear (squares) and nonlinear (dots) CD measured in a pump-probe experiment as a function of the probe wavelength. The pump wavelength is $465 \mathrm{~nm}$. The linear values are measured for a cell thickness of $2 \mathrm{~mm}$; the nonlinear coefficients are given in absolute units $\left(\mathrm{W}^{-1} \mathrm{~cm}^{2}\right)$. The solid lines are the spectra measured using spectrophotometers.

$$
K_{p-p}=\frac{\Delta \beta_{p-p} / \beta_{p-p}}{\Delta \alpha / \alpha_{0}}
$$

It is plotted in Fig. 3 together with the result of a model calculation that we will introduce now.

\section{MODEL CALCULATION}

\section{A. Choice of the model}

We want now to develop a model calculation so as to be able to understand the origin of the light-induced optical activity and to reproduce qualitatively our experimental results.

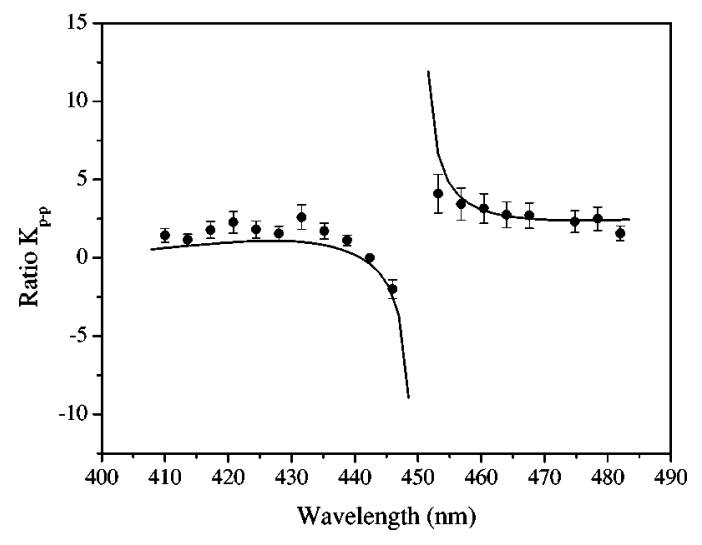

FIG. 3. Ratio $K_{p-p}$ as a function of the probe wavelength (pump wavelength $=465 \mathrm{~nm}$ ). The solid line is a theoretical fit obtained with Eqs. (3.25) and (3.26) with $\delta=1.2$ and $\Omega_{\text {pump }}=0.5 \delta$ (see text). 


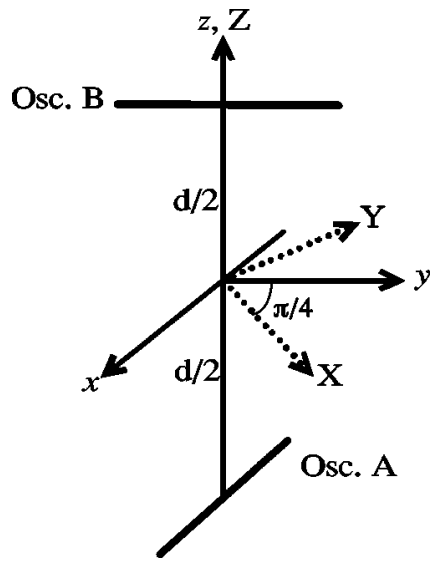

FIG. 4. Representation of the molecule by the two-oscillator model. Oscillator $A(B)$ is along the $x(y)$ direction.

For that purpose, it is worth examining closely the optical transition we are working with. This transition originates in a charge transfer from the metallic ion toward the ligand, and the final state is ${ }^{3} M L C T$ [9]. However, the absorption band corresponds to several close transitions. Our experimental results about the saturation of the absorption [Fig. 2(a)] clearly show that this band is not homogeneous: when pumping on the low-energy side of the transition, the light-induced structure is not centered on the absorption peak, but is shifted on the low-energy side as well. Examining now the origin of the $\mathrm{CD}$, it appears that two transitions are involved, one polarized along the threefold axis of the molecule (symmetry $A_{2}$ ) and another one polarized in the perpendicular plane (symmetry E) [10]. These two transitions are coupled with an in-ligand transition (taking place in the UV region), giving rise to two close opposite $\mathrm{CD}$ bands and to the bisignate spectrum displayed in Fig. 1. One can furthermore calculate that the rotational strengths of the two contributions are equal in magnitude but opposite in sign [10].

However, a detailed description of the electronic levels involved in the absorption and CD spectra is only possible through a complete density-functional calculation [11]. Extending this calculation to include excited states, saturation effects or rapid transfer from ${ }^{1} M L C T$ to ${ }^{3} M L C T$ [9] has never been done and would be very involved. We therefore prefer to introduce a phenomenological model to clarify the experimental results. One way to model our system would be to consider two independent transitions displaying opposite rotational strengths. However, in this description, optical activity would be introduced without any clear relationship with the spatial configuration of the molecule. In particular, implication of these geometrical effects in the nonlinear optical response would not be easy to understand. We therefore choose to describe our system with the degenerate coupled oscillators (Kuhn [12]) model in which optical activity is directly deduced form the molecular configuration. In this model, the molecule is described by two identical oscillators separated by a distance $d$ as represented in Fig. 4. The two oscillators are chosen perpendicular, the first oscillator (labeled $A$ ) parallel to the $x$ direction and the second oscillator $(B)$ parallel to $y$. The two oscillators are furthermore coupled, for example, through dipole-dipole interaction. Note that this description allows us to introduce straightforwardly such effects as the intensity dependence of the coupling parameters, opening up the modeling of light-induced conformational changes.

This model is clearly not the best one to describe quantitatively our molecule but serves as a phenomenological description to allow prediction of the form of the absorption and CD spectra. However, the phenomenological nature of this model is not expected to change dramatically the interpretation of the results since the most important point in our discussions is the frequency position of the absorption and $\mathrm{CD}$ structures and not the absolute magnitudes of the nonlinear effects. Indeed, we have checked that introducing ex abrupto optical activity in two independent transitions gives very similar results, but does not clarify how the optical activity enters the optical nonlinear response.

We come now to the calculation of the linear and nonlinear absorption and CD within this coupled-oscillator model. Let us call $\left|n_{a}\right\rangle\left(\left|n_{b}\right\rangle\right)$ the quantum state of the oscillator $A(B)$. There are two degenerate excited states $\left|1_{a}\right\rangle$ and $\left|1_{b}\right\rangle$ depending on which oscillator is excited. However, these excited states are coupled and, in this basis, the Hamiltonian of the system reads

$$
\left[\begin{array}{ll}
E & V \\
V & E
\end{array}\right],
$$

where $E$ is the energy of each oscillator and $V$ is the coupling energy. The Hamiltonian is not diagonal, and diagonalizing it leads to working in a new basis, obtained from the previous one by a $\pi / 4$ rotation [the new axis system being $X, Y, Z$ (see Fig. 4)]

$$
| \pm\rangle=\frac{1}{\sqrt{2}}\left(\left|1_{a}\right\rangle \pm\left|1_{b}\right\rangle\right) .
$$

In this basis, the Hamiltonian is

$$
\left[\begin{array}{cc}
E+V & 0 \\
0 & E-V
\end{array}\right]
$$

and we deal now with two independent nondegenerate oscillators, as inferred above from our experimental results. We further suppose that each of these two transitions behaves like a two-level system. It is indeed the case for our RuTB samples for which only saturation is observed [13]. In other experimental situations, there can exist an excited-state absorption which is redshifted compared to the ground-state absorption. In these cases, one has to consider the transitions as anharmonic oscillators. Such a calculation with anharmonic oscillators, although not necessary to describe our experimental system, will be addressed in our discussion on magnetic dipolar and electric quadrupolar contributions in Sec. IV.

Finally, we end up with a very simple model consisting of two independent two-level systems. We are interested in calculating the energy transfer between the light and the molecules, introducing linear and third-order nonlinear response. In order to account for the optical activity, we must go be- 
yond the usual local approximation, which means that we cannot neglect the variation of the electromagnetic field across the molecular extension. We will now develop the calculation for such a system, starting by considering only the local response. Nonlocal response will be added later.

\section{B. Local response}

In the case of the local response, the electromagnetic field of the light is supposed constant over the molecular extension. This approximation is most often used, it amounts to considering only the effects of the electric field F. In this approximation, the polarizabilities are defined through the expression of the light-induced dipole $\mathbf{p}$ as

$$
\mathbf{p}=\varepsilon_{0} \alpha^{e e} \mathbf{F}+\varepsilon_{0} \gamma^{e e e e}|\mathbf{F}|^{2} \mathbf{F},
$$

where $\alpha^{e e}$ and $\gamma^{e e e e}$ are the polarizability and the second hyperpolarizability tensors, respectively, whose nonzero components have the following expressions:

$$
\begin{gathered}
\alpha_{X X}^{e e}=\frac{\mu_{01}^{2}}{\varepsilon_{0} m \Gamma^{2}} \frac{1}{\Omega+\delta-i} ; \quad \alpha_{Y Y}^{e e}=\frac{\mu_{01}^{2}}{\varepsilon_{0} m \Gamma^{2}} \frac{1}{\Omega-\delta-i}, \\
\gamma_{X X X X}^{e e e e}=-\frac{\mu_{01}^{4}}{\varepsilon_{0} m \Gamma^{3} \Gamma_{1}} \frac{1}{\left[(\Omega+\delta)^{2}+1\right](\Omega+\delta-i)}, \\
\gamma_{Y Y Y Y}^{e e e e}=-\frac{\mu_{01}^{4}}{\varepsilon_{0} m \Gamma^{3} \Gamma_{1}} \frac{1}{\left[(\Omega-\delta)^{2}+1\right](\Omega-\delta-i)} .
\end{gathered}
$$

In these expressions, we have introduced the relaxation rate for the population $\left(\Gamma_{1}\right)$ and for the coherences $(\Gamma)$, the electric dipole moments of the transitions $\left(\mu_{01}\right)$ (supposed equal for the two transitions), and we note $\Omega \pm \delta$ the detuning between the photon energy and the two transitions, normalized to $\Gamma[\Omega=(E-\hbar \omega) / \hbar \Gamma, \delta=V / \hbar \Gamma]$. From these microscopic expressions, one can deduce the macroscopic susceptibilities $\chi^{e e}$ and $\chi^{e e e e}$ by averaging the above tensors over an isotropic distribution. Both these tensors have only one independent component in that particular case. With their help, one calculates the energy transfer between the light and the molecules which scales as

$$
\Delta=\varepsilon_{0} \operatorname{Im}\left[\mathbf{F}^{*} \cdot \chi^{e e} \cdot \mathbf{F}+\mathbf{F}^{*} \cdot \chi^{e e e e}: \mathbf{F F} \mathbf{F}^{*} \mathbf{F}\right]
$$

Introducing the molecule density $N$, the linear absorption is therefore

$$
\Delta^{(1)}=\frac{N \mu_{01}^{2}}{3 m \Gamma^{2}}|\mathbf{F}|^{2}\left[\frac{1}{(\Omega+\delta)^{2}+1}+\frac{1}{(\Omega-\delta)^{2}+1}\right],
$$

while the nonlinear absorption is

$$
\Delta^{(3)}=-\frac{N \mu_{01}^{4}}{5 m \Gamma^{3} \Gamma_{1}}|\mathbf{F}|^{4}\left[\frac{1}{\left[(\Omega+\delta)^{2}+1\right]^{2}}+\frac{1}{\left[(\Omega-\delta)^{2}+1\right]^{2}}\right]
$$

These two expressions correspond to the expected expressions for two independent two-level systems. Note that due to the high symmetry of the tensors, these expressions are independent of the electromagnetic field polarization.

\section{Nonlocal response}

Let us now proceed to include the nonlocal response of the system, which is necessary to describe optical activity, or more precisely in our case, the dependence of the absorption processes with the handedness of the light polarization.

In this coupled-oscillator system, optical activity arises from the electric dipoles of the two oscillators and there is no need to introduce any magnetic dipoles. We will thus suppose that the optical transitions corresponding to the two oscillators are electric dipole allowed, but magnetic dipole forbidden.

Examining more carefully the origin of optical activity in such system, it is clear that it comes from the fact that the electric fields are not the same at a given time for the two oscillators as one must take into account the effect of the propagation. A very convenient way to translate this into equations is to consider that in the $x y z$ frame, the electric field is

$$
\begin{gathered}
F_{x}(0)-\frac{d}{2} \nabla_{z} F_{x}(0), \\
F_{y}(0)+\frac{d}{2} \nabla_{z} F_{y}(0), \\
F_{z}(0),
\end{gathered}
$$

which we write

$$
F_{i}=F_{i}(0)+c_{i} \nabla_{z} F_{i}
$$

with

$$
c_{x}=-d / 2, c_{y}=+d / 2, c_{z}=0 .
$$

Let us consider now the linear absorption of a given molecule:

$$
\delta^{(1)}=\varepsilon_{0} \operatorname{Im}\left[\mathbf{F}^{*} \cdot \boldsymbol{\alpha}^{e e} \cdot \mathbf{F}\right],
$$

which can be shown to be (assuming implicit summation over repeated indices)

$$
\delta^{(1)}=\varepsilon_{0} \operatorname{Im}\left\{\alpha_{i j}^{e e}\left[F_{i}^{*} F_{j}+c_{i}\left(\nabla_{z} F_{i}^{*}\right) F_{j}+c_{j} F_{i}^{*}\left(\nabla_{z} F_{j}\right)\right]\right\} .
$$

Here we have neglected higher order terms in the gradient of the electric field. Considering a plane wave $\mathbf{F}=\mathbf{A} e^{-i \mathbf{k} \cdot \mathbf{r}}$, we can rewrite Eq. (3.14) as

$$
\delta^{(1)}=\varepsilon_{0}\left[\alpha_{i j}^{e e}+i k_{z} \alpha_{i j z}^{e e \nabla}\right] A_{i}^{*} A_{j},
$$

where we have introduced a nonlocal polarizability

$$
\alpha_{i j z}^{e e \nabla}=\alpha_{i j}^{e e}\left(-c_{i}+c_{j}\right) .
$$


Using Eq. (3.5) and changing the frame from $X Y Z$ to $x y z$, it is easy to calculate $\alpha_{i j z}^{e e \nabla}$ and to average it over an isotropic distribution to obtain the nonlocal susceptibility $\chi^{e e \nabla}$. We suppose now that the electromagnetic wave propagates along a given direction that we call " 3 ." The wave vector is therefore parallel to this direction while the electric field lies in the $(1,2)$ plane. The "nonlocal" linear absorption of this light beam can be calculated as

$$
\Delta^{N L o c}=\varepsilon_{0} \operatorname{Im} \sum_{i, j=1,2} i k\left[\chi_{i j 3}^{e e \nabla} A_{i}^{*} A_{j}\right]
$$

from which we can obtain the expression of the CD. A left $(L)$ or right $(R)$ polarization being characterized by the following electric fields:

$$
\mathbf{A}_{L, R}=\frac{A}{\sqrt{2}}(1, \pm i, 0),
$$

we can apply Eq. (3.17) to get the

$$
\begin{aligned}
C D & \equiv \Delta^{N L o c}(L)-\Delta^{N L o c}(R)=\Delta^{(1)} \\
& =\frac{N \mu_{01}^{2}}{3 m \Gamma^{2}} A^{2} k d\left[\frac{1}{(\Omega+\delta)^{2}+1}-\frac{1}{(\Omega-\delta)^{2}+1}\right] .
\end{aligned}
$$

It is straightforward to extend the above calculation to the third-order response and to define a nonlocal hyperpolarizability

$$
\gamma_{i j k l z}^{e e e \nabla}=\gamma_{i j k l}^{e e e e}\left(-c_{i}+c_{j}-c_{k}+c_{l}\right),
$$

in which case the nonlinear absorption of a given molecule is

$$
\delta^{(3)}=\left[\gamma_{i j k l}^{e e e}+i k_{z} \gamma_{i j k l z}^{e e e e \nabla}\right] A_{i}^{*} A_{j} A_{k}^{*} A_{l} .
$$

It is important to notice that in this description, we do not separate the magnetic dipolar and the electric quadrupolar contributions. Both are a priori mixed in the nonlocal hyperpolarizability, causing there to be no particular symmetry rule concerning the tensor components. We will come back to this problem in Sec. IV, where a comparison between this two-level description with a three-level one will prove to be instructive about the existence of electric quadrupolarization effects in the third-order nonlinear response of chiral liquids.

An analogous treatment of the determination of linear CD allows us to obtain the expression of the nonlinear $\mathrm{CD}$ as

$$
\mathcal{D}=-\frac{N \mu_{01}^{4}}{5 m \Gamma^{3} \Gamma_{1}} A^{4} 2 k d\left[\frac{1}{\left[(\Omega+\delta)^{2}+1\right]^{2}}-\frac{1}{\left[(\Omega-\delta)^{2}+1\right]^{2}}\right] .
$$

Looking at the above equations, it is easy to understand the underlying physics. In case of the usual (i.e., local) response, the linear and nonlinear absorptions of the two oscillators are independent of the handedness of light and add constructively whereas the nonlocal responses, very sensitive to the handedness of light, are opposite for the two oscilla-
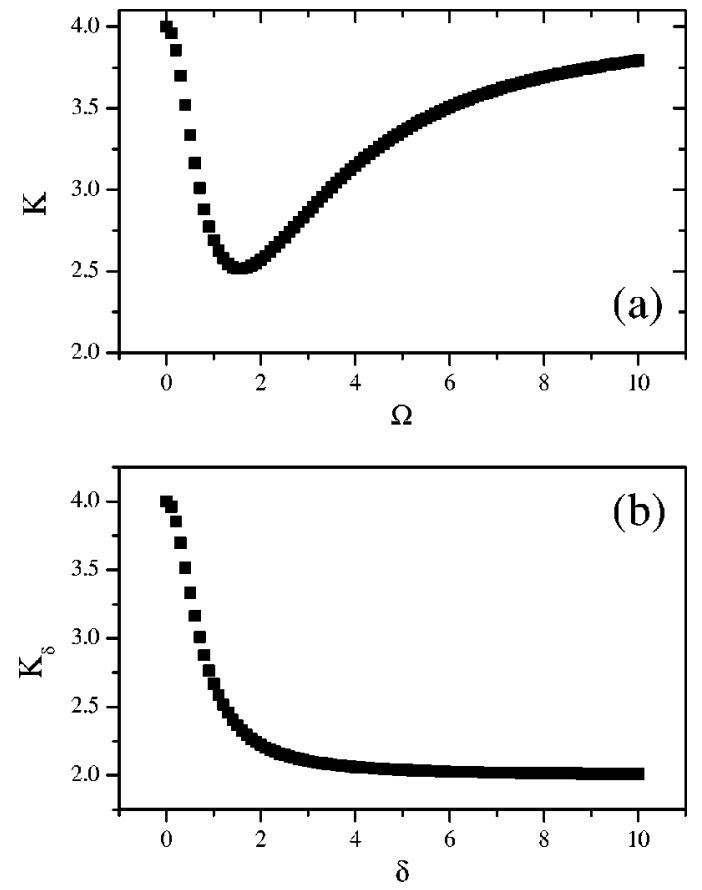

FIG. 5. (a) Ratio $K$ as a function of the detuning $\Omega$ for $\delta=1.2$. (b) Minimum value of $K$ as a function of $\delta$.

tors and must be substracted. This gives the well-known dispersive shape of CD curves (cf. Fig. 1), and from Eq. (3.22), the same is true of the nonlinear CD curves.

From the above expressions, it is possible to express the parameter $K$ that we defined previously

$$
K=\frac{4}{1+\left(\frac{2 \delta \Omega}{\Omega^{2}+\delta^{2}+1}\right)^{2}} .
$$

A plot of $K$ as a function of the normalized detuning is shown in Fig. 5(a) for $\delta=1.2$. Changing the value of $\delta$ does not change the overall shape of the curve but only changes the value of the minimum, which can be calculated as $K_{\delta}$ $=4\left(\delta^{2}+1\right) /\left(2 \delta^{2}+1\right)$ [Fig. 5(b)]. The shape of the curve can be understood as follows. A factor 2 in $K$ can be traced back to the expressions of the nonlocal polarizabilities: $2 c_{i}$ 's are present in the linear term [Eq. (3.16)] while $4 c_{i}$ 's are involved in the nonlinear one [Eq. (3.20)]. It is a result of the numbering of the gradient terms involved in the process. The other factor 2 in $K$ (at $\Omega=0$ ) comes from the partial cancellation in the $\mathrm{CD}$ bands which are not the same for the linear and the nonlinear terms. However, when $\Omega$ gets closer to $\delta$, i.e., when one gets closer to resonance with one of the two transitions, the relative weights of the two transitions change and so do the interference effects. Note that if a unique transition was considered, one would obtain a value of 2 for $K$.

From this discussion, it is clear that the shape of the curve $K(\Omega)$ is very sensitive to the configuration of the transitions involved in the process. Let us now compare these calculations with the experimental results of Sec. II. First of all, we see that the overall agreement is quite good. The experimen- 


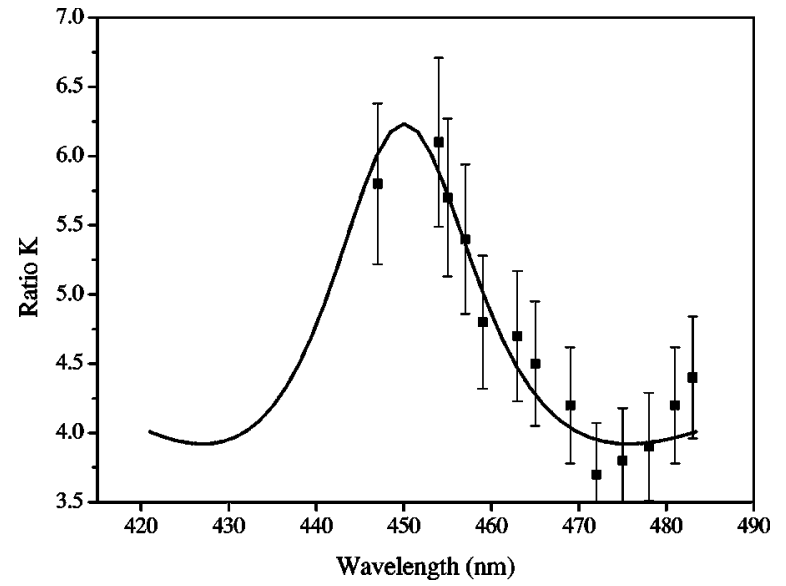

FIG. 6. Fitting of the experimental data displayed in Fig. 1 with Eq. (3.23) for $\delta=1.2$.

tal and model values of $K$ are of the same order and the tendency of the experimental curve (Fig. 1) mimics the theoretical one [Fig. 5(a)]. We can be more quantitative. In order to estimate the parameter $\delta$, we remark that the experimental ratio between the maximum and the minimum values of $K$ is around 0.63 , as determined from the theory for $\delta$ $=1.2[$ cf. Fig. 5(b)]. We have, therefore, chosen this value to fit our data. Results of the fits are displayed in Fig. 6, where we have plotted the parameter $K$. The solid lines correspond to the calculations and the dots to the experimental data, the agreement is quite good. In particular, the shapes of the curves are very well reproduced. This indicates that our system cannot be represented by a unique two-level system but, as far as optical activity is concerned, necessitates at least two two-level systems. This simple description provides a good explanation for the processes involved in the linear and nonlinear optical activity of these molecules. The only discrepancy is between the absolute values of $K$ which are larger experimentally than theoretically by a factor of 1.5. This likely comes from the fact that the metal-to-ligand charge-transfer transition in RuTB involves, in reality, more than two transitions [11] and that a model with more than one pair of two-level systems would likely be more appropriate. Nevertheless, the shape of the CD and $K$ curves is mainly determined by the existence of such pairs.

\section{Pump-probe configuration}

It is straightforward to extend the above calculation to the pump-probe experiments. The main difference is in the hyperpolarizability expressions. For a pump linearly polarized along $X$, the relevant components are

$$
\begin{aligned}
\gamma_{X X X X}^{e e e e} & =-\frac{\mu_{01}^{4}}{\varepsilon_{0} m \Gamma^{3} \Gamma_{1}} \frac{1}{\left[\left(\Omega_{\text {pump }}+\delta\right)^{2}+1\right](\Omega+\delta-i)}, \\
\gamma_{Y Y X X}^{\text {eeee }} & =-\frac{\mu_{01}^{4}}{\varepsilon_{0} m \Gamma^{3} \Gamma_{1}} \frac{1}{\left[\left(\Omega_{\text {pump }}-\delta\right)^{2}+1\right](\Omega-\delta-i)},
\end{aligned}
$$

where we have introduced the detuning for the pump photons. The nonlinear absorption and CD now read

$$
\begin{aligned}
\Delta_{p-p}^{(3)}= & -\frac{\mu_{01}^{4}}{5 m \Gamma^{3} \Gamma_{1}} A^{4}\left[\frac{1}{\left[\left(\Omega_{\text {pump }}+\delta\right)^{2}+1\right]\left[(\Omega+\delta)^{2}+1\right]}\right. \\
& \left.+\frac{1}{\left[\left(\Omega_{\text {pump }}-\delta\right)^{2}+1\right]\left[(\Omega-\delta)^{2}+1\right]}\right], \\
\mathcal{D}_{p-p}=- & \frac{\mu_{01}^{4}}{5 m \Gamma^{3} \Gamma_{1}} A^{4} 2 k d\left[\frac{1}{\left[\left(\Omega_{\text {pump }}+\delta\right)^{2}+1\right]\left[(\Omega+\delta)^{2}+1\right]}\right. \\
- & \left.\frac{1}{\left[\left(\Omega_{\text {pump }}-\delta\right)^{2}+1\right]\left[(\Omega-\delta)^{2}+1\right]}\right]
\end{aligned}
$$

From these expressions, for comparison to the experimental results, we obtain the coefficient $K_{p-p}$ as a function of the probe frequency for a fixed pump frequency. In Fig. 3, we have plotted the results for the same parameters as Fig. 6 ( $\delta=1.2$ ) together with the experimental data. $\Omega_{\text {pump }}$ is chosen equal to $0.5 \delta$. Here again, the agreement is quite good, except for an overall factor of 1.5. Note that this factor is the same as utilized in Fig. 6. This confirms that the lightinduced CD's in the one- or two-beam experiments originate from the same mechanisms, well accounted for by our two two-level system model.

\section{MAGNETIC DIPOLAR VS ELECTRIC QUADRUPOLAR CONTRIBUTIONS}

Our calculations allow us to clarify somewhat the role of magnetic dipolar and electric quadrupolar contributions in the third-order nonlinear response of an isotropic liquid of chiral molecules. Indeed, the nonlocal contributions introduced above may be separated in these two contributions from a formal point of view. On the other hand, it is well known that electric quadrupolar effects cancel out when isotropically averaged for linear optics. What about these contributions in the third-order nonlinear response? Very general arguments indicate that such quadrupole contributions should exist in nonlinear optics [2]. We will now address this question in view of our models.

First of all, we rewrite Eq. (3.21) as

$$
\begin{aligned}
\delta^{(3)}= & \gamma_{i j k l}^{e e e e} A_{i}^{*} A_{j} A_{k}^{*} A_{l}+\gamma_{i j k z l}^{e e e \nabla e} A_{i}^{*} A_{j} A_{k}^{*} \nabla_{z} A_{l} \\
& +\gamma_{i j z k l}^{e e \nabla e e} A_{i}^{*} A_{j} \nabla_{z} A_{k}^{*} A_{l}+\gamma_{i z j k l}^{e \nabla e e e} A_{i}^{*} \nabla_{z} A_{j} A_{k}^{*} A_{l} \\
& +\gamma_{z i j k l}^{\nabla e e e e} \nabla_{z} A_{i}^{*} A_{j} A_{k}^{*} A_{l}
\end{aligned}
$$

so as to keep track of which gradient out of the four possible ones comes into play in the absorption process. Comparison with Eqs. (3.20) and (3.21) shows for example, that

$$
\gamma_{i j k z l}^{e e e \nabla e}=c_{l} \gamma_{i j k l}^{e e e e}
$$

Similar relations exist for the other coefficients. From these expressions, it is straightforward to introduce the elec- 
tric quadrupolar and magnetic dipolar hyperpolarizabilities by symmetrization and antisymmetrization of $\gamma_{i j k z l}^{e e e \nabla e}$,

$$
\begin{aligned}
& \gamma_{i j k z l}^{e e e Q}=\frac{1}{2}\left[\gamma_{i j k z l}^{e e e \nabla e}+\gamma_{i j k l z}^{e e e \nabla e}\right], \\
& \gamma_{i j k m}^{e e e m}=\frac{1}{2}\left[\gamma_{i j k z l}^{e e e \nabla e}-\gamma_{i j k l z}^{e e e \nabla e}\right] .
\end{aligned}
$$

( $m$ obeying the relation $\varepsilon_{z l m}=1$ with $\varepsilon$ : Levi-Civita tensor). Note that the $\gamma_{i j k z l}^{e e e Q}$ is symmetrical with respect to the interchange of the last two indices. Here again, one obtains similar relations for the other hyperpolarizabilities. From these microscopic quantities, one can obtain the isotropically averaged susceptibilities $\chi^{e e e Q}$ and $\chi^{e e e m}$ and access the relative contributions of the electric quadrupolarization and of the magnetic polarization in the third-order nonlinear response.

Performing this calculation for our previous model (twocoupled two-level systems), one determines that $\chi^{e e e Q}=0$ whereas $\chi^{e e e m} \neq 0$, which means that there is no quadrupolar contribution present. This is, however, not a general rule, but a particular feature of two-level systems. We had already demonstrated this point from a general quantum point of view in [6].

In order to verify that indeed quadrupolar effects can contribute to the third-order nonlinear response, even though these do not in linear optics, we now consider another model. We suppose that the two coupled oscillators of our model do not behave as two two-level systems, but as two anharmonic oscillators. This changes the expression of the local hyperpolarizability tensor which has now eight nonzero components instead of two and which read, if we suppose that the energy difference between the first and the second excited states is close to that between the first excited state and the ground state:

$$
\begin{gathered}
\gamma_{X X X X}^{\text {eeee }} \propto \frac{2}{\left[(\Omega+\delta)^{2}+1\right](\Omega+\delta-i)^{2}}, \\
\gamma_{X X Y Y}^{\text {eeee }}=\gamma_{X Y Y X}^{\text {eeee }} \propto \frac{1}{\left[(\Omega-\delta)^{2}+1\right](\Omega+\delta-i)^{2}}, \\
\gamma_{Y Y X X}^{\text {eeee }}=\gamma_{Y X X Y}^{\text {eeee }} \propto \frac{1}{\left[(\Omega+\delta)^{2}+1\right](\Omega-\delta-i)^{2}}, \\
\gamma_{Y Y Y Y}^{\text {eeee }} \propto \frac{2}{\left[(\Omega+\delta)^{2}-1\right](\Omega-\delta-i)^{2}} .
\end{gathered}
$$

From these expressions, it is straightforward to carry out the calculation of the nonlocal hyperpolarizabilities and to deduce the electric quadrupolar and magnetic dipolar nonlinear susceptibilities. Carrying out all the above calculations, we determine that the results of Sec. III do not change qualitatively. However, the important point for our present discussion is that we calculate that $\chi^{\text {eee } Q}$ as well as $\chi^{\text {eeem }}$ are both nonzero and have the same order of magnitude. This demon- strates that one cannot, in general, neglect the quadrupolarization contributions in the third-order nonlinear response.

This feature is an illustration of the potential of nonlinear optics to investigate more thoroughly molecular properties. For example, one can consider molecules which do not have any magnetic-dipole-allowed transitions but possess electricquadrupole-allowed ones. As is well known, these molecules will not display any optical activity once istotropically dispersed in a solvent. However, nonlinear optical activity is allowed and it is quite possible that these molecules show a light-induced circular dichroism.

\section{CONCLUSION}

This paper is devoted to a close examination of the wavelength dependence of the nonlinear circular dichroism in a chiral salt of RuTB. A one-beam experiment using a tunable laser source is used to measure the dispersion of the NLCD which displays a strong variation across the linear CD structure. On the other hand, a pump-probe experiment with a tunable probe allows us to determine the nonlinear CD spectrum. These features are then theoretically interpreted through the development of a calculation based on a twocoupled two-level system model which reproduces satisfactorily the experimental data when the nonlocality of the light-matter interaction is considered. All the third-order nonlinear effects derive from a saturation of the two optical transitions, the effects of which add constructively for the usual absorption effects while they interfere destructively as far as (linear or nonlinear) optical activity is concerned. Our calculation also allows us to address the question of the contribution of electric quadrupolarization to the nonlinear response of isotropic liquids of chiral molecules. Studying the nonlocal response for a two-anharmonic-oscillator system, we isolate such contributions, as expected from general arguments [2].

These experiments open up more techniques for the investigation of ultrafast conformational changes in molecules. Indeed, it is clear from the coupled-oscillator model that the $\mathrm{CD}$ is very sensitive to geometrical parameters such as the distance between the oscillators or their relative orientation. The time-resolved CD signal that can be measured in a pump-probe experiment on varying the time delay between the two beams will therefore be very sensitive to lightinduced changes in these parameters. This technique should allow such changes to be followed in real time. It is however important to keep in mind that purely electronic effects can induce a nonlinear circular dichroism, independent of the conformational changes under study. These effects are clearly seen in this paper dealing with a ruthenium salt that does not undergo any noticeable conformational change but nevertheless displays a large nonlinear $\mathrm{CD}$. The correct agreement between our calculations and our experiments clearly shows that the electronic nonlinear optical activity is well understood and can be quantified through the measurement of the nonlinear absorption, making it easier to sort out the electronic and the conformational contributions to the time-resolved $\mathrm{CD}$. These experiments should provide very sensitive techniques for the study of time-resolved conformational changes in chiral molecules. 
[1] S. A. Akhmanov and V. I. Zharikov, Pis'ma Zh. Eksp. Teor. Fiz. 6, 644 (1967) [JETP Lett. 6, 137 (1967)]; N. I. Koroteev, in Frontiers in Nonlinear Optics, edited by H. Walther, N. I. Koroteev, and M. Scully (Institute of Physics, Bristol, 1993), pp. $228-239$.

[2] G. Wagnière, J. Chem. Phys. 77, 2786 (1982).

[3] H. Ashitaka, Y. Yohoh, R. Shimizu, T. Yokozawa, K. Morita, T. Suehiro, and Y. Matsumoto, Nonlinear Opt. 4, 281 (1993).

[4] R. Cameron and G. C. Tabisz, Mol. Phys. 90, 159 (1997).

[5] H. Mesnil and F. Hache, Phys. Rev. Lett. 85, 4257 (2000).

[6] F. Hache, H. Mesnil, and M. C. Schanne-Klein, Phys. Rev. B 60, 6405 (1999).

[7] H. Mesnil, M. C. Schanne-Klein, F. Hache, M. Alexandre, G.
Lemercier, and C. Andraud, Chem. Phys. Lett. 338, 269 (2001).

[8] G. M. Gale, G. Gallot, F. Hache, and R. Sander, Opt. Lett. 22, 1253 (1997).

[9] A. Yeh, C. V. Shank, J. K. McCusker, Science 289, 935 (2000).

[10] A. Rodger and B. Nordén, Circular Dichroism and Linear Dichroism (Oxford University Press, Oxford, 1997), p. 86.

[11] C. Daul, E. J. Baerends, and P. Vernooijs, Inorg. Chem. 33, 3538 (1994).

[12] W. Kuhn, Z. Phys. Chem. Abt. B 14, 29 (1929).

[13] N. H. Daumrauer, G. Cerullo, A. Yeh, T. R. Boussie, C. V. Shank, and J. K. McCusker, Science 275, 54 (1997). 